Malte Klein

\title{
Durch Forschendes Lernen kennenlernen, was Digitalität mit Geschichte macht
}

Ein Kommentar zum Projektbericht Forschungswerkstatt zur Erstellung einer Digitalen Edition von Bianca Frohne und Swantje Piotrowski ${ }^{1}$

\section{Einführung: Didaktische Potenziale des Universitätsprojekts}

Das Projekt Forschungswerkstatt zur Erstellung einer Digitalen Edition von Bianca Frohne und Swantje Piotrowski berührt - wie man bereits dem Veranstaltungstitel entnehmen kann - mehrere Bereiche der Geschichtsdidaktik. Es geht

1. um das Forschende Lernen an Universitäten (s. Abschnitt ,Zum Forschenden Lernen'),

2. um den Einsatz des pädagogischen Lernwerkstattkonzepts (s. Abschnitt ,Zur Lernwerkstatt'),

3. um den großen Bereich des, Lernens mit digitalen Medien (s. Abschnitt ,Zum Lernen mit digitalen Medien') und

4. um die berufliche Qualifizierung von Geschichtsstudierenden, die nicht nur auf Lehramt studieren (s. Abschnitt ,Zu den Chancen der Berufsqualifizierung').

\section{Zum Forschenden Lernen: Erfahren, wie aufwändig Wissenschaft ist}

Im Projekt Forschungswerkstatt zur Erstellung einer Digitalen Edition haben sich Bianca Frohne und Swantje Piotrowski verdienstvoll zur Aufgabe gesetzt, sehr alte, schwer lesbare Quellen gemeinsam mit Studierenden zu erschließen: Es handelt sich um Selbstzeugnisse, Briefe und Leichenpredigten zwischen 1300 und 1800, die sich mit dem betroffen machenden Themenfeld ,Tod und Sterben', insbesondere mit dem Schwerpunkt Sterbeanleitungen und ,guter Tod' von Schwerkranken auseinandersetzen. Dies ist für die Studierenden neben der Erschließung und Erlernung des Umgangs mit der Digitalisierung zugleich fachlich anspruchsvoll, da auch gefordert wird, über das jeweils zeitlich vorherrschende Memorialwesen in Verbindung mit dem durchaus differenzierten

1 Kommentierter Beitrag: https://dx.doi.org/10.38072/2703-0784/p12.

>> Das Werk ist unter der Creative-Commons-Lizenz Namensnennung - Weitergabe unter gleichen Bedingungen 4.0 International veröffentlicht. Den Vertragstext finden Sie unter: https://creativecommons.org/licenses/by-sa/4.0/deed.de. Bitte beachten Sie, dass einzelne, entsprechend gekennzeichnete Teile des Werks von der genannten Lizenz ausgenommen sein bzw. anderen urheberrechtlichen Bedingungen unterliegen können. 
Wandel der gesellschaftlichen Mentalitäten zu reflektieren. ${ }^{2}$ Zentrales Element ist somit für die Projektleiterinnen neben der fachlichen, berufsqualifizierenden und kompetenzorientierten Ausrichtung die Verzahnung von Theorie und Praxis unter den Bedingungen des Forschenden Lernens. ${ }^{3}$

Und das durchgeführte Projekt hat durchaus das Potenzial, den zentralen Leitideen des Forschenden Lernens, die 2019 im Sammelband Forschendes Lernen in den Geisteswissenschaften aufgeführt werden, ${ }^{4}$ gerecht zu werden:

Die Studierenden erschließen fachlich und methodisch ihre eigenen Sinnzusammenhänge durch die Selbstorganisation ihres Lernprozesses (Prinzip des Selbstlernansatzes): Techniken zur Entzifferung von Handschriften und schwer lesbaren Texten werden eigenständig eingeübt, ebenso wird der Umgang der Edition-Software nach eigenem Ermessen erprobt und vertieft, wobei auch eigene Aufzeichnungen diese individuellen Lernwege sichtbar machen. Die Offenheit der durch die Seminarleitung gestellten Aufgaben trägt dazu bei, dass die Studierenden diesen Selbstlernansatz entsprechend verfolgen können. ${ }^{5}$

Dem Einführen in die Enkulturation des Forschens (Prinzip des Forschens), die in der Literatur als Einüben der Wissenschaftlichkeit in zirkulären Prozessen beschrieben wird, wird durch das Aufteilen in wiederkehrende Vertiefens-/Übungs- und Auswertungsphasen Rechnung getragen. Überdies gehört zur Enkulturation des Forschens ein Transfer der elaborierten Erkenntnisse über die Vorstellungen zum ,guten Tod' auf geschichtswissenschaftliche Inhalte. Da die Teilnehmenden hierdurch auch in die Lage versetzt werden, ggf. eigene Metatheorien zu formulieren und diese ausgehend von Ergebnissen der Geschichtswissenschaft weiterzuentwickeln, besteht perspektivisch die Möglichkeit, unmittelbar die Dynamik des Wissenszuwachses innerhalb eines Fachgebiets zu erfahren. Damit erfüllt Frohnes und Piotrowskis Ansatz weitere Prinzipien des Forschenden Lernens, namentlich das der (Selbst-)Reflexion und das des forschenden Habitus. ${ }^{6}$

Gerade die anspruchsvolle Transformation der Handschriften in das digitale Editionsformat kann für neue Wege der Hypothesenbildung umso hilfreicher sein, da digitalisierte Quellenformate einen anderen statistischen Zugriff ermöglichen. Begriffsgeschichtlich ließe sich so beispielsweise die Häufigkeit des Vorkommens bestimmter

2 Vgl. z. B. Peter Dinzelbacher, Europa im Hochmittelalter. 1050-1250, eine Kultur- und Mentalitätsgeschichte. Darmstadt 2003 (Kultur und Mentalität), $96 \mathrm{f}$.

3 Margrit E. Kaufmann/Ayla Satilmis/Harald A. Mieg, Einleitung: Forschendes Lernen in den Geisteswissenschaften, in: Margrit E. Kaufmann/Ayla Satilmis/Harald A. Mieg (Hrsg.), Forschendes Lernen in den Geisteswissenschaften. Konzepte, Praktiken und Perspektiven hermeneutischer Fächer. Wiesbaden 2019, 1-20.

4 Vgl. Kaufmann/Satilmis/Mieg, Einleitung (wie Anm. 3) Vgl. dabei insbesondere auch Andreas Bihrer/Stephan Bruhn/Fiona Fritz, Forschendes Lernen in Geschichtswissenschaft und Geschichtsstudium, in: Kaufmann/Satilmis/Mieg, Forschendes Lernen (wie Anm. 3), 105-124.

5 Zur Relevanz offener Aufgabenstellung in Szenarien des selbstorganisierten Lernens vgl. z. B. Birgit Landherr/Manfred Bürger, SOL - selbstorganisiertes Lernen. Systematische Kompetenzentwicklung in einer komplexen Welt. 3. Aufl. Baltmannsweiler 2019, 23.

6 Vgl. Kaufmann/Satilmis/Mieg, Einleitung (wie Anm. 3), $7 \mathrm{f}$. 
Worte untersuchen. Hieraus könnten sich neue Fragestellungen ergeben. Die Teilnehmenden erfahren somit die Auswirkungen neuerer wissenschaftlicher Trends, hier ließen sich Bezüge zum ,Digital Turn` oder zur ,Digital Literacy` herstellen (Prinzip der Partizipation an der Wissenschaft). ${ }^{7}$

Damit verbunden ist der Kompetenzzuwachs, eine weitere Leitidee des Forschenden Lernens: Die Studierenden werden in einem breiten Feld qualifiziert, da sie einen Einblick bekommen, wie der Prozess der Quellensuche, -entzifferung, -übersetzung und -interpretation ablaufen kann. Zugleich üben sie die Quellenkritik, sie können praktisch erfahren, was der hermeneutische Zirkel eigentlich bedeutet. Dies gilt nicht nur interpretatorisch, auch das Entziffern von schwer lesbaren Handschriften erfordert so manche wiederkehrende Neuüberprüfung. Frohne und Pietrowski arbeiten mit bislang wenig gesichteten und wenig erschlossenen Quellen, wodurch Forschung sich umso authentischer darstellt.

Wertvoll ist auch der Transfer, nämlich der Versuch quellenbasiert allgemein gültige, zusammenfassende Aussagen über die Vergangenheit zu finden: So können sich die Projektteilnehmer*innen gefragt haben, wie aussagefähig die vereinzelten Briefe und Leichenpredigten über die tatsächliche Praxis der Durchführung von Sterbevorbereitungen in der frühen Neuzeit sind. Die Studierenden haben die Möglichkeit, diese Ereignisse zu rekonstruieren und herauszuarbeiten, wie es - frei nach Ranke - tatsächlich gewesen sein könnte. Entsprechend können Erfahrungen zur Theoriebildung gesammelt werden. Mit anderen Worten: Die Studierenden erkennen vermutlich auch, wie sich induktive Aussagen über die Phänomene des Sterbens und der Sterbevorbereitung in Mittelalter und Neuzeit nur mit Vorsicht entwickeln lassen. Zum hermeneutischen Zirkel gehört, dass man Entscheidungen treffen muss: Die Projektleiterinnen thematisieren genau diese Frage: Welche Information ist in den Quellen zur Weiterverarbeitung relevant, welche nicht? Aus dieser subjektiven und beobachterabhängigen Informationsauswahl werden dann Aussagen über das damalige Geschehen getroffen - perspektivisch lässt sich damit der Konstruktcharakter von Geschichte verdeutlichen.

Vielleicht lernen die Teilnehmenden darüber hinaus, dass bei der per se begrenzten Quellenauswahl aller Ereignisse der Vergangenheit das gewisse Unbehagen über die gewonnene Erkenntnis bleiben muss, da quellengestützte Informationen nie vollständig sein können und zugleich auch im jeweiligen Kontext und im Spiegel der (sich immer weiter entwickelnden) Fachliteratur ausgelegt und überprüft werden müssen. ${ }^{8}$

7 Vgl. Gerben Zaagsma, On Digital History, in: bmgn - Low Countries Historical Review 128, 2013, 3-29.

8 Gerade hier ergibt sich das Problem des hermeneutischen Zirkels: Der Kontext erschließt sich nur über die Daten der Quellen oder - deduktiv - durch die (zeitgenössische) Literatur. Verabsolutierende Aussagen sind beim Versuch der (Re-) Konstruktion kaum möglich. 
Dieser Einblick in die Haltung des/der Forschenden (Prinzip des Forscher*innenhabitus) ist im Hinblick auf die Schulpraxis insofern wertvoll, als dass sie angehenden Lehrkräften Begründungen liefern, mit deren Hilfe sie Schüler*innen zeigen können, wie einerseits mühselig aber andererseits auch nachvollziehbar der geschichtswissenschaftliche Weg der Erkenntnis ist. Forschendes Lernen kann zudem auch ganz pragmatisch verdeutlichen, dass es vielerlei Methoden, Ansätze und Wissenschaftsschulen gibt, die für sich plausibel und schlüssig den Anspruch auf Wissenschaftlichkeit erheben, aber, im Sinne des Methodenpluralismus, durchaus zu unterschiedlichen Ergebnissen kommen können (Prinzip der Diversität des Forschenden Lernens), zugleich lässt sich damit auch die geschichtsdidaktische Multiperspektivität verdeutlichen. ${ }^{9}$ Auf die Lehrer*innenbildung bezogen bedeutet das, dass Studierende lernen könnten, in ihrer späteren Rolle als Geschichtslehrkraft bei hitzigen Diskussionen über den historischen Wahrheitsgehalt eine gewisse Gelassenheit zu entwickeln.

\section{Zur Lernwerkstatt: Geringe Abgrenzung zum Forschenden Lernen?}

Das Projekt trägt im Titel den Begriff ,Forschungswerkstatt: Was sind die pädagogischdidaktischen Hintergründe? Der Begriff der Forschungswerkstätten erlebte seine Hochkonjunktur Anfang der 2000er Jahre. ${ }^{10}$ Verwandte Ideen, die im Bereich Erziehung mit dem Werkstattbegriff zusammenhängen, sind z. B. Lernwerkstätten oder die ,pädagogische Werkstattarbeit؛ Diese Ideen reichen zurück in die Reformpädagogikbewegung der 1920er Jahren. ${ }^{11}$ In Kiel gab es an der seit 2003 aufgelösten Erziehungswissenschaftlichen Fakultät eine pädagogische Werkstatt, vermutlich mitbegründet vom Pädagogen Waldemar Pallasch, der in Publikationen mitunter auch die Grundlagen pädagogischer Werkstattarbeit definierte: Lernwerkstätten sollen eine ,anregende Lernumgebung schaffen, d. h. sie sollen durch die bereitgestellten Materialien Aktivitäten der Lernenden hervorrufen, sie sollen zu weiterführenden Fragestellungen der Lernsubjekte führen. Eine Abgrenzung gegenüber Formaten wie Workshops bestehe bei Werkstätten dahin gehend, dass sich nicht allein Expert*innen austauschen, sondern gewissermaßen

\footnotetext{
9 Zu den einzelnen Erkenntnistheorien und Wissenschaftsschulen gerade im Themenfeld der Wissensaneignung sowie im Bereich der allgemeinen Didaktik lieferte zuletzt Benner eine umfassende Übersicht: Vgl. Dietrich Benner, Umriss der allgemeinen Wissenschaftsdidaktik. Grundlagen und Orientierungen für Lehrerbildung, Unterricht und Forschung. Weinheim 2020, 76-198. Zur Problematik der Letztbegründung von Wissenschaftstheorien vgl. insbesondere Wolfgang Deppert, Kritik der normativen Wissenschaftstheorien. Wiesbaden 2017 (Theorie der Wissenschaft, Bd. 3), 15-20.

10 So zumindest die quantitative Auswertung bei Google Books. Vgl. Google Books Ngram Viewer, Begriff ,Forschungswerkstatt' 1800-2019, 2021, https://books.google.com/ngrams/graph?content=Forschungswerkstatt\&year_start=1800\&year_ end=2019\& corpus=31 \&smoothing=3\# (letzter Zugriff: 27.5 .2021 ).

11 Vgl. Knut Vollmer, Fachwörterbuch Kita. Schnelle Zugänge für pädagogische Fachkräfte, München 2021, 254. Das Konzept der Lernwerkstätten ist im Zuge den Reformschulen der 1920er Jahre entstanden, greift sogar - wenn man Lehrwerkstätten der Kunstschulen miteinbezieht - in die Zeit der Kunstgewerbebewegung des 19. Jahrhunderts. Vgl. Malte Klein, Das Kunstgewerbemuseum Flensburg. Konzeption und Funktionen eines Museums im Kaiserreich. Kiel 2007, 29.
} 
multiprofessionelle Teams. ${ }^{12}$ Eine Diversität bezüglich der Fähigkeiten der Akteur*innen ist durchaus erwünscht, offene Lernsettings sollen eine ertragreiche Zusammenarbeit begünstigen. ${ }^{13}$ So lassen sich Überschneidungen mit den Merkmalen des Forschenden Lernens (s. Abschnitt ,Zum Forschendes Lernen') feststellen: Offenheit der Aufgabenstellung, Arbeitsteilung, Verquickung Theorie/Praxis sowie intensive, begleitende Betreuung der Teilnehmenden, was bei Frohne und Piotrowski entsprechend umgesetzt wird (s. auch Prinzipien des Coaching und der Kommunikation beim Forschenden Lernen). Der Wechsel der Sozialformen beim durchgeführten Kieler ,Lehr-Lern-Versuch“, die Einzelarbeitsphasen, die Zerlegung der Digitalisatsproduktion in ,Teilwerkstätten“, die im Bericht beschriebenen Stationswechsel und die gemeinsame Reflexion mit den Studierenden sowie die Evaluation runden das Projekt gelungen ab. Man kann annehmen, dass der Begriff der Lernwerkstatt sich vornehmlich auf den Ort und auf die Qualität der bereitgestellten Lernmaterialien bezieht, in denen meistens etwas Neues, etwas Exploratives erprobt wird. ${ }^{14}$

Nähert man sich aus der Domäne Geschichte den Werkstätten, so kommt man nicht umhin, auf die sozialhistorischen Geschichtswerkstätten hinzuweisen, die aus der schwedischen Bewegung ,Grabe-wo-du-stehst' entstanden sind. ${ }^{15}$ Es handelt sich dabei allerdings um basisdemokratische, emanzipatorisch ausgerichtete, häufig frei organisierte, regionalgeschichtliche Forschungsinitiativen, die nicht so sehr mit Universitäten verbunden sind wie die pädagogischen Lernwerkstätten. ${ }^{16}$ Ihr Ziel ist es daher nicht, vornehmlich Lernmaterialien zum freien Ausprobieren bereit zu stellen. Wobei es durchaus Geschichtswerkstätten gibt, die dann Lernorte, z. B. in der Nähe von Gedenkstätten entwickeln, in denen Quellen wie Fotografien, Texte usw. von Gruppen untersucht werden können. ${ }^{17}$

\footnotetext{
12 Vgl. Waldemar Pallasch/Heino Reimers, Pädagogische Werkstattarbeit. Eine pädagogisch-didaktische Konzeption zur Belebung der traditionellen Lernkultur. Weinheim/München 1990 ((Pädagogisches Training), 92. Die Pädagogische Werkstatt befand sich zuletzt in der Olshausenstraße 75, Gebäude 3, Raum 053 (Untergeschoss), 24118 Kiel. Vgl. Christian-AlbrechtsUniversität zu Kiel, Berufsbegleitende Weiterbildung „Coaching” an der Kieler Uni, 2021, https://www.uni-kiel.de/de/universitaet/detailansicht/news/berufsbegleitende-weiterbildung-coaching-an-der-kieler-uni (letzter Zugriff: 27.5.2021).

13 Vgl. Liva Enders/Stephanie Winter, "Das hatte ich mir anders vorgestellt”. Studierende und Dozierende im Spannungsfeld zwischen Theorie und Praxis - Die Hochschullernwerkstatt als Forschungsraum, in: Kathrin Kramer/Dietlinde Rumpf/Miriam Schöps/Stephanie Winter (Hrsg.), Hochschullernwerkstätten - Elemente von Hochschulentwicklung? Ein Rückblick auf 15 Jahre Hochschullernwerkstatt in Halle und andernorts 2020, 129-144.

14 Vgl. auch Vollmer, Fachwörterbuch Kita (wie Anm. 11), 254.

15 Vgl. Sven Lindqvist/Manfred Dammeyer (Hrsg.), Grabe, wo du stehst. Handbuch zur Erforschung der eigenen Geschichte. Bonn 1989.

16 Vgl. Joachim Szodrzynski (Hrsg.), Geschichtswerkstätten gestern - heute - morgen. Bewegung! Stillstand. Aufbruch? 1. Aufl. München i.e. Ebenhausen, Hamburg 2004 (Hamburger Zeitspuren, Bd. 2.), 13, 24. Sie leisten meist unabhängig von Institutionen Aufklärungsarbeit z. B. über benachteiligte Gruppen und wenden sich dabei der lokalen Alltags- und Mikrogeschichte zu. Diese Geschichtswerkstätten haben sich seit den 1970er Jahren zunehmend in Vereinen organisiert, die noch heute einen regen Austausch mit Kommunen, Schulen und Volkshochschulen pflegen. Siehe z. B. das Hamburger Netzwerk der Geschichtswerkstätten. Vgl. Geschichtswerkstätten Hamburg, 2021, https://geschichtswerkstaetten-hamburg.de/html/ ueber_uns__.html (letzter Zugriff: 27.5.2021). Siehe auch in Braunschwieg ,Arbeitskreis Andere Geschichte' e.V, Arbeitskreis Andere Geschichte: „Über uns", 2021, http://www.andere-geschichte.de/uber-uns/ (letzter Zugriff: 27.5.2021).
}

17 Vgl. z. B. die Gedenkstätte Schillstraße der Braunschweiger Geschichtswerkstatt. Vgl. ,Arbeitskreis Andere Geschichte' (wie Anm. 16, letzter Zugriff: 27.5.2021). 


\section{Zum Lernen mit digitalen Medien: Profilierungschancen für Geschichte?}

Im Beitrag von Frohne und Piotrowski wird deutlich, dass der Kompetenzzuwachs im Umgang mit digitalen Medien einen hohen Stellenwert hat und zwar nicht nur dahingehend, dass Technologie zur Effizienzsteigerung eingesetzt wird. Das Digitale spielt selbst eine tragende Rolle, wie das historische Wissen über den ,guten Tod' konstruiert und vermittelt wird: Die Studierenden überführen die Quellenwörter in einen Hypertext und pflegen ihre Übersetzungen in die (vorher festgelegten) Strukturen von Datenbanken ein. Dazu gehören Metadaten, formale Aussagen über Quellen sowie Entitäten und die Fähigkeit der klaren Codierung. Es entstehen folglich nicht allein Digitalisate, elektronische (Hyper-)Texte, sondern auch Metaquellen, die ggf. eine Neudefinition von traditioneller Quellenkritik verursachen: Werden diese erhobenen Daten, wie Frohne und Piotrowski andeuten, auch im Internet oder in einem Forschungsintranet vernetzt, so müssen Historiker*innen nicht nur die etablierte klassische Quellenkritik, sondern auch Ansätze der ,geschichtswissenschaftlichen Datenkritik' kennen. Schon beim Erschließen relevanter digitaler Daten für die Geschichtswissenschaft stellen sich epistemologische Fragen, die die Triftigkeit und die Integrität, Authentizität und Historizität der untersuchten Objekte betreffen. ${ }^{18}$

All das kann - wie Sam Wineburg empirisch gezeigt hat - gravierende Folgen für die Wissensaneignung haben. Die rein digitale Erschließung im Internet, bei der man zwangsläufig mit Scoring und Algorithmen konfrontiert wird, haben - nach Wineburgs Studie - Historiker*innen trotz ihrer Ausbildung nicht ohne weiteres in die Lage versetzt, digitale Quellen richtig zu deuten. ${ }^{19}$ Frohnes und Piotrowskis Ansatz kann bei gründlicher Reflexion vielleicht auch dazu beitragen, dass Studierende die Chancen und Grenzen digitaler Medien in ihrem fachlichen Einsatz schärfer wahrnehmen..$^{20}$

Das ist ein besonderer Mehrwert des Projekts Forschungswerkstatt zur Erstellung einer Digitalen Edition, da hiermit domänenspezifische mediendidaktische Fähigkeiten entwickelt werden. Mit anderen Worten: Es können medienbezogene fachliche Kompetenzen trainiert werden, wie das Ausloten der Chancen und Grenzen einer Technologie (z. B. des Oxygen-Editors) oder das Ausloten der Gefahrenquellen bei der Erstellung von Meta-Quellen-Daten usw. Auch das Reflektieren über die möglichen epistemologischen und methodologischen Folgen bei der Herstellung und Nutzung digitaler Daten in der Forschung kann zu einem fachlichen Kompetenzzuwachs führen.

\footnotetext{
18 Ferner kann der ungeprüfte Einsatz von KI-Software wie z. B. die automatische Handschriftenerkennung Transkibus der ReadGenossenschaft hinsichtlich der Validität der Daten fehlerhafte Transkriptionen begünstigen. Vgl. READ COOP, Transkribus | KI-gestützte Plattform zur Erkennung von handschriftlichem Text, 2021, https://readcoop.eu/de/transkribus/ (letzter Zugriff: 27.5.2021). 19 Vgl. Sam Wineburg, Warum historische Kompetenzen für die Auswertung von digitalen Quellen nicht ausreichend sind, in: Sebastian Barsch/Andreas Lutter/Christian Meyer-Heidemann (Hrsg.), Fake und Filter. Historisches und politisches Lernen in Zeiten der Digitalität. 2019 (Wochenschau Wissenschaft), 105-120.
}

$20 \mathrm{Vgl}$. Andreas Fickers: Update für die Hermeneutik. Geschichtswissenschaft auf dem Weg zur digitalen Forensik?, in: Zeithistorische Forschungen. Studies in Contemporary History 1, 2020, https://doi.org/10.14765/zzf.dok-1765. 
Darüber hinaus werden gleichsam fachunabhängigere medienbezogene Kenntnisse der Informatik, wie das Grundwissen von Hard- und Software sowie anderes technologisches Konzeptwissen, wie z. B. kollaboratives Arbeiten, Charakteristika des Hyptertextes/Algorithmen/Datenbanken mit vermittelt. ${ }^{21}$

Die Möglichkeit, solche Ansätze zur Kompetenzaneignung durch das Projekt zu verfolgen, ist nicht nur relevant im Sinne der Geschichtslehrpläne und der Lehrer*innenausbildung - wie im Projektbericht aufgeführt - sondern betrifft alle Studierende: Universitäten werden explizit im bundesweiten Konzeptpapier zur Medienbildung der Kultusministerkonferenz (KMK) ,Bildung in der digitalen Welt' (2016) als „Orte zur Entwicklung, Erprobung und Anwendung von Formen und Methoden der digitalen Lehre sowie zur Erforschung der individuellen und gesellschaftlichen Folgen der Digitalisierung“ deklariert. Wenn alle Schüler*innen gemäß der KMK-Standards seit dem Schuljahr 2018/19 grundlegende Kompetenzen der Medienbildungen erhalten sollen, entsteht entsprechender Bedarf für Studierende, dass diese Kompetenzen in der Hochschule weiter entwickelt werden. ${ }^{22}$

Und hier - wie bereits angedeutet - liefert das durchgeführte Projekt von Frohne und Piotrokowski einen ertragreichen Anknüpfungspunkt zu geschichtsdidaktischen Reflexionen über die domänenspezifische Verwendung von digitalen Medien. Für die Schulpraxis, insbesondere in der Fach- und Unterrichtsentwicklung, kann die Frage gestellt werden, inwieweit sich Geschichte gegenüber anderen Fächern im Lernen mit

21 Über Kompetenzen in der Medienbildung vgl. z.B. Lena von Kotzebue/Ulrike Franke/Florian Schultz-Pernice/Monika Aufleger/Birgit J. Neuhaus/Frank Fischer, Kernkompetenzen von Lehrkräften für das Unterrichten in einer digitalisierten Welt, in: merz - medien + erziehung, Zeitschrift für Medienpädagogik, 2017, 65-74. Es gibt auch andere Formen der Modellierung der digitalen Kompetenzen, prominent ist das TPACK-Modell, das zuletzt von Burghard Barte und Horst Schilling in der Geschichtsdidaktik eingeordnet wurde. Vgl. Burghard Barte/Horst Schilling, Historisches Lehren im Bedingungsgefüge von Geschichtskultur, Lehr-Lernkultur und digitale Massenmedien, in: Sebastian Barsch/Burghard Barte (Hrsg.), Motivation - Kognition Reflexion. Schlaglichter geschichtsdidaktischer Professionsforschung. Frankfurt a. M. 2021 (Geschichtsunterricht erforschen, Bd. 12), 178-197.

22 Es handelt sich um die Kompetenzbereiche als integrativer Bestandteil der Fachcurricula aller Fächer: „1. Suchen, Verarbeiten und Aufbewahren, 2. Kommunizieren und Kooperieren. 3. Produzieren und Präsentieren, 4. Schützen und sicher Agieren, 5. Problemlösen und Handeln und 6. Analysieren und Reflektieren". Vgl. Strategie ,Bildung in der digitalen Welt', 2021, https:// www.kmk.org/aktuelles/artikelansicht/strategie-bildung-in-der-digitalen-welt.html (letzter Zugriff: 26.5.2021). Recht zeitgleich wurde auch die Dagstuhl-Erklärung publiziert, Resultat eines Treffens von „Expert_innen aus der Informatik und ihrer Didaktik, der Medienpädagogik, der Wirtschaft und der Schulpraxis", die ebenfalls den fächerintegrativen Ansatz wie bei der KMK berücksichtigt. Vgl. Gesellschaft für Informatik, Bildung in der digitalen vernetzten Welt. Eine gemeinsame Erklärung der Teilnehmerinnen und Teilnehmer des Seminars auf Schloss Dagstuhl -Leibniz-Zentrum für Informatik GmbH, 2016, https://gi.de/fileadmin/GI/Hauptseite/Themen/Dagstuhl-Erkla_rung_2016-03-23.pdf (letzter Zugriff: 26.5.2021).

Auch die Europäische Kommission hat für das Digital Living aller Bürger*innen grundsätzliche digitale Kompetenzbereiche herausgearbeitet, die recht ähnlich sind, nämlich 2017 im DigComp 2.1: The Digital Competence Framework for Citizens with eight proficiency levels and examples of use. Die übergeordneten Kompetenzbereiche lauten: Information and data literacy, Communication and collaboration, Digital content creation, Safety und Problem solving. Vgl. European Commission. Joint Research Centre, DigComp 2.1. The digital competence framework for citizens with eight proficiency levels and examples of use 2017. Diese Kompetenzmodelle erfahren in vielen Bundesländern und Bildungseinrichtungen Modifikationen. Lehrerinstitute wie das schleswig-holsteinische IQSH arbeiten an Medienkompetenzrahmen für die Lehrerbildung. Verschiedene Vorschläge zur Reformierung der universitären Lehre werden derzeit erarbeitet und z.T. schon publiziert, vgl. z. B. Marcel Capparozza/ Gabriele Irle, Digitale Kompetenzen von Lehrerausbildenden. Ein Überblick über empirische Modelle, in: Kai Kaspar/Michael Becker-Mrotzek/Sandra Hofhues/Johannes König/Daniela Schmeinck (Hrsg.), Bildung, Schule, Digitalisierung. Münster, New York 2020, 414-420. Vgl. auch Sarah Genner, Zehn Thesen zu Bildung und Digitalisierung, in: Marko Demantowsky/Gerhard Lauer/Robin Schmidt/Bert te Wildt (Hrsg.), Was macht die Digitalisierung mit den Hochschulen? 2020, 141-152. Vgl. Julia Suckut/Sabrina Förster, Ein Kategoriensystem zur digitalisierungsbezogenen Beschreibung von schulischen und hochschulischen Lehr-Lernumgebungen, in: Kaspar/Becker-Mrotzek/Hofhues/König/Schmeinck, a.a.0., 433-438. 
digitalen Medien besonders fachspezifisch auszeichnet. ${ }^{23}$ Das Lehr-Lern-Projekt zeigt indes sehr plastisch, wie im Fach Geschichte digitale Daten generiert werden und wie man sie fachspezifisch einsetzen kann. Das sind nützliche Erfahrungen, um den fachlichen Bereich der Medienbildung für den Geschichtsunterricht zu profilieren. ${ }^{24}$ Die Mitarbeit an der Digitalisierung der Leichenpredigten und Sterbeanleitungen sowie die Reflexion darüber, was es bedeuten könnte, solche Quellen öffentlich der Allgemeinheit zur Verfügung zu stellen, zeigt auf sehr transparente Weise den Studierenden, wie Forschung, Publikation und Präsentation Implikationen für die gesellschaftliche Öffentlichkeit erzeugen können.

Darüber hinaus ergibt sich die Möglichkeit, das Lernen mit digitalen Medien auf die Geschichtskultur zu beziehen. Frohne und Piotrowski deuten die Erstellung eines Blogs an. Auch das Mitmachen bei der Blogerstellung kann Entstehungsbedingungen sowie beabsichtigte und nicht beabsichtigte Wirkungen im weiten Feld der Geschichts- oder Erinnerungskultur einer Gesellschaft deutlich machen.

Konkretisiert werden könnte auch die außerschulische, geschichtsdidaktische Perspektive z. B. von digitalen Citizen-Science-Projekten. So könnten durch Onlineveröffentlichungen der Digitalisate über den ,guten Tod`z. B. in frei zugänglicher Onlinepräsentation Mechanismen der virtuellen Kulturvermittlung mit thematisiert werden. Nicht zuletzt in ihrem Potenzial des Gegenwartsbezugs: Das aufwühlende Thema ,Tod und Sterben', insbesondere die zeitgenössischen Handlungsempfehlungen zum ,guten Tod', sind aufgrund der Sterbehilfedebatten des Bundestags auch heute noch von hoher Aktualität und politischer Brisanz. ${ }^{25}$

\footnotetext{
23 Denn auch in anderen Fächern - wie Deutsch, Sprachen, Gesellschaftswissenschaften, vermitteln Lehrkräfte Methoden, um digitale Informationen bestimmter Internetdarstellungen und Narrative von Nachrichten oder gesellschaftlichen Zuständen zu dekonstruieren. Ferner: Der Mehrwert durch den Einsatz digitaler Medien im Geschichtsunterricht ist empirisch noch nicht geklärt. Vgl. Alfons Kenkmann, Kommentar. „Womit?" (Digitale) Medien des historischen Lernens, in: Thomas Sandkühler/Charlotte Bühl-Gramer/Anke John/Astrid Schwabe/Markus Bernhardt (Hrsg.), Geschichtsunterricht im 21. Jahrhundert. Eine geschichtsdidaktische Standortbestimmung. Göttingen 2018, 487-492.

24 Ulf Kerber hat auch ausgehend von der Podiumsdiskussion des Historikertags 2012 festgehalten, dass die Themen wie "neue Quellenformen" (E-Mail/Blogs/Chats usw.), Text Mining in historischen Textkorpora, neue elektronische Verfahren der Archivarbeit, qualitative Text- und Datenanalyse, historische Netzwerkforschung, Geographic Information Systems (GIS), Spatial History und neue Wege der wissenschaftlichen Kommunikation durchaus genuin fachspezifische Herausforderungen der Geschichte und ihrer Didaktik seien. Entstehungsbedingungen digitaler Produkte, Artefakte oder Kommunikationsmedien müssen geschichtswissenschaftlich aber auch geschichtsdidaktisch immer mitgedacht werden. Vgl. Ulf Kerber, Medientheoretische und medienpädagogische Grundlagen einer "Historischen Medienkompetenz", in: Marko Demantowsky/Christoph Pallaske (Hrsg.), Geschichte lernen im digitalen Wandel. Berlin 2015, 106-131. Erst kürzlich wurde im aktuellen Band Digitale Mediävistik und der deutschsprachige Raum auf die Komplexität der digitalen Editionsarbeit hingewiesen: „Editionen sind nicht mehr als Einzelunternehmen zu denken, sondern verlangen nach neuen Formen interdisziplinären und kollaborativen Arbeitens." Vgl. Roman Bleier/Franz Fischer u. a., Digitale Mediävistik und der deutschsprachige Raum. Themenheft: Das Mittelalter $24,2019,1,1-12$.

25 Vgl. Deutscher Ärzteverlag GmbH, Redaktion Deutsches Ärzteblatt, Bundestag will über neues Sterbehilfegesetz diskutieren, 2020, https://www.aerzteblatt.de/nachrichten/119427/Bundestag-will-ueber-neues-Sterbehilfegesetz-diskutieren (letzter Zugriff: 27.5.2021).
} 


\section{Zu den Chancen der Berufsqualifizierung von Geschichtsstudierenden außerhalb der Lehrer*innenausbildung}

Auf dem Arbeitsmarkt erzeugen digitale Innovationen massive Veränderungen und Transformationen der Beschäftigungsfelder, als Trend zeichnen sich zunehmend neue Formen der Arbeitsorganisationen, der Produktions- und Dienstleistungsflexibilisierung ab. Merkmale seien nach den Prognosen des Ifo-Instituts das gleichzeitige Ausprobieren des Neuen sowie die Effizienzsteigerung bestehender Prozesse mit massiven Auswirkungen auf (Infra-)Struktur-, Personal- und Kulturebene. ${ }^{26}$ In dieser Gemengelage stellt sich die Frage, wie Historiker*innen arbeiten und zukünftig arbeiten werden.

In der Geschichtswissenschaft ist das Auswerten von Archivalien das Kerngeschäft. Häufig verbringen Wissenschaftler*innen örtlich gebunden Tage bis Wochen damit, Aktenberge, Nachlasse, Sammlungen von Handschriften oder kirchliche Register zu durchforsten, meist verbunden mit der Hoffnung, brauchbare Daten für das eigene Forschungsthema zu finden. Analoge Quelleneditionen und Übersetzungen können das bereits erleichtern, umso wertvoller sind entsprechende digitale Lösungen. ${ }^{27}$ Völlig ortsunabhängig, frei von Archivöffnungs- und -aushebungszeiten werden Einblicke in Quellen möglich. Digitale Archive, z. B. Urkunden als Digitalisate machen schwer zugängliche Quellen also für alle, sowohl für Forscher*innen als auch Lai*innen, sichtbar.

Ökonomisch betrachtet haben wir es im Zuge der Digitalisierung mit einem innovativen Dienstleistungsmarkt zu tun, der immer ausgeklügeltere digitale Modi zur Forschungsunterstützung anbietet, sei es öffentlich finanziert oder durch Angebote von Privatunternehmen. Und diese innovativen Dienstleistungen haben ihre Stärken und Schwächen. Denn der Effizienz und schnellen Verfügbarkeit, den neuen, eigenständigen Auswertungs- und Forschungspotenzialen durch KI-Einsatz, steht meist das wenig nachvollziehbare Überführen von Daten in Cloudspeicher ohne ausreichende Gewährleistung von Datenschutz gegenüber (siehe auch Abschnitt ,Zum Lernen mit digitalen Medien`). ${ }^{28}$

26 Vgl. Oliver Stettes/Melanie Arntz/Terry Gregory/Ulrich Zierahn/Katharina Dengler/Daniel J. Veit/Werner Eichhorst/Ulf Rinne, Arbeitswelt 4.0. Wohlstandszuwachs oder Ungleichheit und Arbeitsplatzverlust - was bringt die Digitalisierung?, in: Ifo-Schnelldienst 70, 2017, 3-18. Vgl. auch Felix Stalder, Kultur der Digitalität. Berlin 2016, $12 f$.

27 Die Unterteilung in digitale und analoge Medien wird in der Medientheorie diskutiert, digitale Medien gelten zum einen als "medientechnisch-mediales Apriori", da eben die technische Entwicklung ausschlaggebend für ihre Existenz ist. Auf der anderen Seite sind alle Medien per se analog ausgerichtet auf die Nutzenden. Digitale Informationen werden für den menschlichen, „analogen" Körper über Schnittstellen sichtbar gemacht. Somit müssen digitale Medien immer einen analogen Output berücksichtigen. Vgl. Jens Schröter/Alexander Böhnke, Analog/Digital - Opposition oder Kontinuum? Zur Theorie und Geschichte einer Unterscheidung. s.l. 2004 (Medienumbrüche, Bd. 2) Zum generellen Wandel der Medien vgl. auch Tanja Gnosa, Historisches oder mediales Apriori? Versuch einer terminologischen Rejustierung, in: Le foucaldien 3, 2017, 1-32.

28 Cloudfunktionen bei Literaturdatenbanken wie z. B. bei dem Update Citavi 6 können dazu führen, dass Nutzer*innen gegen europäisches Datenschutzrecht verstoßen. Vgl. https://www.sub.uni-goettingen.de/fileadmin/media/texte/benutzung/Literaturverwaltung/Citavi_Datensicherung_Handout_20190326.pdf (letzter Zugriff: 27.5.2021). Auf die Spitze getrieben stellt sich in der immer stärker expandierenden digitalen Welt die philosophische Frage, inwieweit der Mensch Technik überhaupt (noch) beherrschen kann. Vgl. Leonhard Dobusch, Ohnmacht trotz technologischer Potenz. Pfade informationstechnologischer Selbstentmachtung, in: Dorina Gumm/Monique Janneck/Roman Langer/Edourd J. Simon (Hrsg.), Mensch - Technik - Ärger? Zur Beherrschbarkeit soziotechnischer Dynamik aus transdisziplinärer Sicht. Berlin 2008 (Arbeitsgestaltung - Technikbewertung - Zukunft, Bd. 19.), 103-118. 
Das berufsqualifizierende Methodenrepertoire der Historiker*innen muss somit den professionellen Umgang mit diesen digitalen Angeboten umfassen. Es geht um den Prozess des Überführens, des Transkribierens alter Schriftstücke in digitale Formate und um die Auswertung und Erschließung digitalisierter Quellen für die Forschung. Es geht zugleich auch um das technologische und rechtliche Wissen, wie man diese Daten schützt und sichert. ${ }^{29}$ Zudem sollten Historiker*innen in der Lage sein, digitale Quellen und digital dargebotene Informationen richtig zu rezipieren und korrekt $\mathrm{zu}$ interpretieren.

Kurzum: Die Studierenden des Projektes Forschungswerkstatt zur Erstellung einer Digitalen Edition von Bianca Frohne und Swantje Piotrowski arbeiten also recht authentisch im Tätigkeitsfeld potenzieller Beschäftigungsszenarien, die sich im öffentlichen und privaten Umfeld des Archivwesens und der Bibliotheken ergeben könnten. ${ }^{30}$ Das Projekt bietet damit die Chance, berufsqualifizierende Inhalte im Geschichtsstudium mit zu integrieren und zwar auch für diejenigen, die außerhalb des Lehramtes ihren Weg gehen wollen.

\section{Autor}

\section{Malte Klein}

Lehrbeauftragter für Didaktik der Geschichte an der Christian-Albrechts-Universität zu Kiel, abgeordneter Medienberater am Institut für Qualitätsentwicklung an Schulen Schleswig-Holsteins und Lehrer am Kieler Ernst-Barlach Gymnasium. Seine Forschungsschwerpunkte sind Digitale Bildung, Nationalsozialismus und Bildungsgeschichte.

klein@histosem.uni-kiel.de

29 Vgl. Georg Eckert/Thorsten Beigel, Historisch Arbeiten. Handreichung zum Geschichtsstudium. Göttingen 2019 (UTB Geschichte, Studienratgeber, Bd. 5039), 79f. Vgl. auch Peter Haber, Zeitgeschichte und Digital Humanities, Version: 1.0, in: Docupedia-Zeitgeschichte, 24.9.2012, http://dx.doi.org/10.14765/zzf.dok.2.269.v1.

30 Vgl.z. B. Schleswig-Holstein, Büchereizentrale, Karriere, 2021, https://www.bz-sh.de/karriere (letzter Zugriff: 27.5.2021). Vgl. auch Archivar/in (Diplom), 2019, https://www.schleswig-holstein.de/DE/Fachinhalte/A/ausbildung_landsh/Berufe/archivarln_diplom.html (letzter Zugriff: 27.5.2021). 